\title{
Riqueza y distribución de Hílidos (Hylidae: Anura) en zona de bosques pluvial tropical en el departamento del Chocó, Colombia
}

\author{
Wealth and distribution of Hylides (Hylidae: Anura) in a tropical \\ pluvial forest area in the Chocó Department, Colombia
}

\author{
Lizeth Palacios-Rodriguez ${ }^{1 *}$ Biol; Jhon Rengifo-Mosquera ${ }^{1}$ Ph.D; \\ Magleyvi Roa Panesso ${ }^{1}$ Biol; Yakeline Palacios Asprilla ${ }^{1}$ Biol.
}

'Universidad Tecnológica del Chocó, Facultad de Ciencias Básicas y Exactas, Programa de Biología, Grupo de Investigación en Herpetología, Colombia.

\section{KEYWORDS:}

Hylides;

conservation;

habitat;

primary forest;

secondary forests.

\section{ABSTRACT}

In the present study, the richness and distribution of Hylides in the tropical rain forest area were determined, plant coverings were established (primary and secondary forest) for a better analysis of the group in the central area of the department of Chocó. For the estimation of the wealth we used the method of surveys by visual encounters (Visual Encounter Survey "VES"), registering a total of 137 ind., Grouped into five genera and eight species of Hílidos. There was a difference in the distribution of the species according to the vegetation cover, registering better values in secondary forest ( $s=8$ and $n=103)$; in the community the best represented genera were Dendropsophus, Scinax and Boana who were present in both coverages, and in specific term Boana boans and Smilisca phaeota. In the use of habitat, significant difference was found in the vertical positions $(P=1.34)$, as well as the substrates evaluated $(P=1.66)$ these differences are evidenced in soil position and substrates branches. The results revealed that the Hílidos frequent the secondary forests because of their heterogeneity that holds the ideal conditions and ecological requirements that these species require for their development.

\section{RESUMEN}

El presente estudio se determinó la riqueza y distribución de Hílidos en zona de bosque pluvial tropical, se establecieron coberturas vegetales (bosque primario y secundario) para un mejor análisis del grupo en la zona centro del departamento del Chocó. Para la estimación de la riqueza se utilizó el método de relevamientos por encuentros visuales (Visual Encounter Survey "VES"), registrando un total de 137 ind., agrupados en cinco géneros y ocho especies de Hílidos. Se encontró diferencia en la distribución de las especies según las coberturas vegetales, registrando mejores valores en bosque secundario $(s=8$ y $n=103)$; en la comunidad los géneros mejores representados fueron Dendropsophus, Scinax y Boana quienes estuvieron presentes en ambas coberturas, y en término especifico Boana boans y Smilisca phaeota. En el uso de hábitat, se encontró diferencia significativa en las posiciones verticales $(P=1.34)$, al igual que los sustratos evaluados $(P=1.66)$ estas diferencias están evidenciadas en posición suelo y sustratos ramas. Los resultados revelaron que los Hílidos frecuentan los bosques secundarios por su heterogeneidad y que ostenten las condiciones idóneas y requerimientos ecológicos que estas especies precisan para su desarrollo. 
Palacios-Rodriguez et al - Riqueza y distribución (Hylidae: Anura) en bosques pluvial tropical

\section{INTRODUCCIÓN}

Los anuros son un grupo de vertebrado que se encuentra bien representados en todos los ecosistemas, lo que los hace más abundante y diversificado de los anfibios vivientes. Las especies de este grupo se encuentran en hábitats acuáticos, terrestres, fosoriales y arborícolas en prácticamente todos los continentes (HALLIDAY y ADLER 2007). En Colombia se encuentran 14 familias de anuros, entre ellas, la familia Hylidae con 124 especies aproximadamente, representadas en seis subfamilias (Batrachia.com).

Los Anuros por su parte, son particularmente sensibles a la fragmentación de los bosques debido a sus características fisiológicas y comportamentales, sumado a factores ambientales, como la temperatura, la precipitación y la humedad relativa del aire que determinan su distribución ecológica y geográfica (OSORNO, 1999). En las últimas dos décadas un gran número de investigadores se ha dedicado a estudiar las alarmantes disminuciones poblacionales y las extinciones de anfibios ocurridas en diversas partes del mundo (ANGULO, 2002; BLAUSTEIN et al., 1994; PECHMANN et al., 1991; STUART et al., 2006,). Entre las causas identificadas que explican estos fenómenos globales, la más citada es la destrucción de los hábitats (YOUNG et al., 2004; LIPS et al., 2005), seguida por los cambios climáticos (POUNDS y CRUMP, 2004; POUNDS y PUSCHENDORF, 2004), y la introducción de especies exóticas (KIESECKER et al., 2001).

Uno de los problemas ambientales más relevantes a escala global es la reducción de la diversidad biológica asociada a los efectos de pérdida, fragmentación y transformación del hábitat como consecuencia de las actividades humanas (HANKEN, 1999; KNUTSON et al., 1999, 2000), en estas actividades la instalación de asentamiento humano, significa la pérdida total de hábitat para muchas especies; sin embargo, para otras implica el surgimiento de nuevos ambientes adecuados para la colonización y desarrollo de sus poblaciones. De modo que, la integridad del hábitat en el que se desenvuelven los anfibios del departamento del Chocó, está siendo amenazada por esta serie de acciones, factores y procesos que directa o indirectamente ocasionan la degradación o sustitución de los ecosistemas naturales según (VARGAS y BOLAÑOS, 1999b).

Es importante resaltar que los anfibios representan una importante fracción de organismos necesarios para el mantenimiento de los sistemas biológicos y debido a sus condiciones fisiológicas y requerimientos ecológicos, son muy sensibles a los cambios en los ecosistemas y altamente dependientes de las condiciones ambientales (VARGAS y CASTRO, 1999; RUEDA-ALMONACID et al., 2004).

Son pocos los estudios o investigaciones realizadas en el departamento de Chocó que permitan conocer el estado actual de comunidades, especialmente grupos de alta sensibilidad a los cambios ambientales producto de impactos generados por las acciones antrópicas, por tal razón, el presente trabajo se realizó con el fin de determinar la riqueza y distribución de estas especies en diferentes coberturas vegetales en zonas de bosques pluvial tropical en la zona centro del departamento del Chocó, con la finalidad de contribuir al conocimiento de las poblaciones de Hílidos para generar estrategias de conservación.

\section{MATERIAL Y MÉTODOS}

Área de estudio: Las zonas de muestreos se localizan en las Ilanuras aluviales, colinas bajas y piedemonte cercanos al valle del río Atrato (municipio de Quibdó, departamento del Chocó), donde se concentra la mayor pluviosidad del andén del Pacífico (Chocó Biogeográfico) y donde las formaciones selváticas se encuentran entre las más ricas del mundo (CUATRECASAS, 1958; FORERO y GENTRY, 1989).

El área de estudio se localiza entre las coordenadas geográficas $5^{\circ} 00^{\prime}-6^{\circ} 45^{\prime} \mathrm{N}$ y 77015'-76030'O. El régimen de precipitación es de tipo bimodal-tetraestacional, con un período de mayor concentración de lluvias entre abril y octubre y una época 
de menor concentración desde noviembre hasta marzo (POVEDA et al., 2004). La precipitación anual es de $8.558 \mathrm{~mm}$ con un promedio mensual de 395,5 mm (RANGEL$\mathrm{CH}$. y LOWY, 1993). Las comunidades vegetales de esta zona aparecen detallas en (CUATRECASAS 1958; SUÁREZ-NAVARRO et al., 1984). Los datos fueron tomados en campo. Los limites climáticos generales que presentan un promedio de lluvia anual superior a $8.000 \mathrm{~mm}$ y una temperatura media superior a $24^{\circ} \mathrm{C}$. (Tabla 1 , Figura 1 ).

Trabajo de Campo: Se seleccionaron dos zonas de muestreos según la cobertura vegetal, la primera denominada bosque primario, que presenta como características la bajas o nulas perturbaciones humanas, u otros disturbios durante períodos que exceden el largo normal de la vida de los árboles maduros de 60 a 80 años según FAO (ANÓN. 1982c) y bosque secundario como una vegetación de carácter sucesional (proceso de regeneración natural del bosque) que se desarrolla sobre tierras, donde el bosque original ha sido destruido por actividades humanas. "Su grado de recuperación dependerá mayormente de la duración e intensidad del uso anterior por cultivos agrícolas o pastos, así como de la proximidad a fuentes semilleros para recolonizar el área alterada" SMITH et al., (1997).

Trabajo de campo: Los muestreos se realizaron entre los meses marzo y junio, empleando un esfuerzo de muestreo de 240 horas/persona, con una duración de seis días en cada cobertura vegetal y se realizaron réplicas en los cinco puntos de muestreo. En el bosque primario se realizaron observaciones cerca de cuerpos de agua con vegetación ribereña, árboles y arbustos; en cuanto al bosque secundario, se observó en

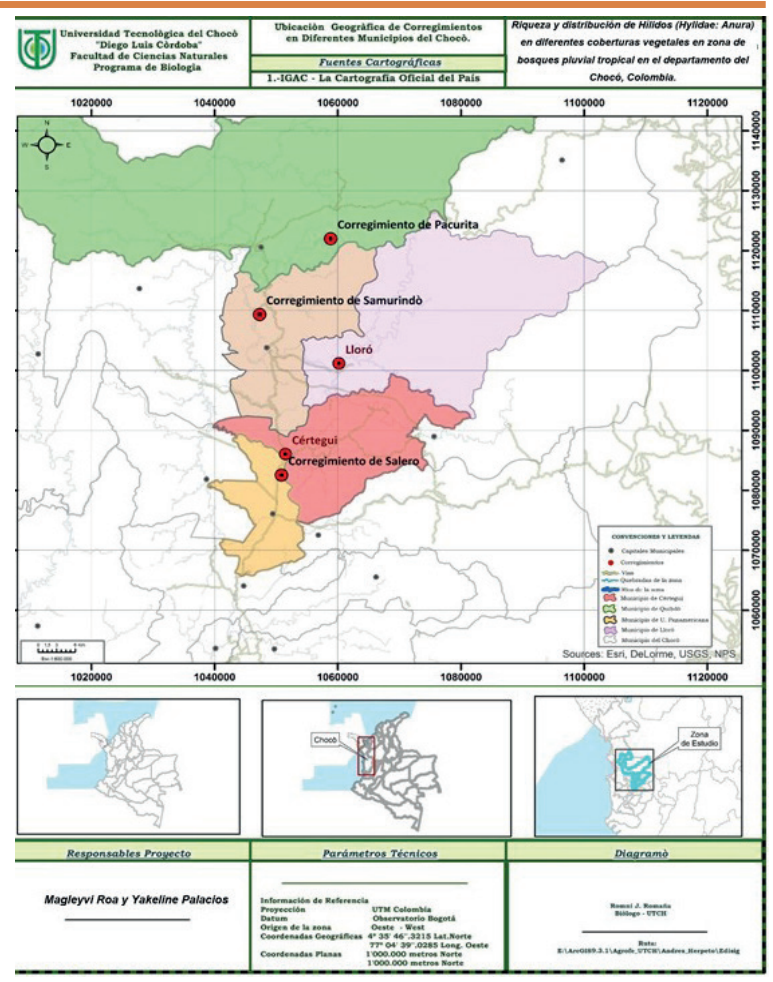

Figura 1.Ubicación Geográfica del Área de Estudio, en los Cinco Municipios de la Zona Centro del Departamento del Chocó, Colombia.

corrientes de agua superficialmente de baja calidad, en sitios con poca cubierta vegetal o con algún grado de transformación, aplicando el método de búsqueda por relevamiento visuales (siglas en ingles VES), técnica utilizada para determinar la riqueza de especies en un área y estimar las abundancias relativas de las especies en una comunidad (CRUMP y SCOTT, 1994), la cual consiste en la observación directa de las especies en los diferentes hábitats (árboles, arbustos, bordes de camino, troncos y cuerpos de agua loticos y lenticos).

Tabla 1. Ubicación de las zonas de muestreos en un bosque pluvial tropical, centro del departamento del Chocó.

\begin{tabular}{lcccccc}
\hline \multicolumn{1}{c}{ "Puntos de Muestro" } & Localización & T ${ }^{\circ} \mathbf{C}$ & \% & \multicolumn{2}{c}{ Altura } & PP(mm) Zona de Vida \\
\hline Unión Panamericana "Salero" & $5^{\circ} 22^{\prime} \mathrm{N}, 76^{\circ} 36^{\prime} \mathrm{W}$ & 28 & 90 & 100 & 7.600 & $\mathrm{bp}-\mathrm{T}$ \\
Certegüi "Recta Larga" & $5^{\circ} 41^{\prime} 41^{\prime \prime} \mathrm{N}, 76^{\circ} 39^{\prime} 40^{\prime \prime} \mathrm{W}$ & 28 & 95 & 43 & 7.000 & $\mathrm{bp}-\mathrm{T}$ \\
Lloró "CMUTCH" & $5^{\circ} 30^{\prime} 37^{\prime \prime} \mathrm{N}, 76^{\circ} 33^{\prime} 15^{\prime \prime} \mathrm{W}$ & 28 & 85 & 48 & 10.000 & $\mathrm{bp}-\mathrm{T}$ \\
Atrato "Samurindó" & $5^{\circ} 35^{\prime} 15^{\prime \prime} \mathrm{N}, 76^{\circ} 39^{\prime} 15^{\prime \prime} \mathrm{W}$ & 28 & 91,8 & 30 & 8.000 & bp-T \\
Quibdó "Urbana y Suburbana" & $5^{\circ} 43^{\prime} 13^{\prime \prime} \mathrm{N}, 76^{\circ} 37^{\prime} 40^{\prime \prime} \mathrm{W}$ & 26,5 & 87 & 47 & 12.000 & bp-T \\
\hline
\end{tabular}


Palacios-Rodriguez et al - Riqueza y distribución (Hylidae: Anura) en bosques pluvial tropical

La determinación taxonómica se realizó con base en las guías de campo de (PÁEZ et al., 2002, RENJIFO y LUNDBERG 1999) y artículos científicos (CUENTAS et al., 2002, MENDOZA, 2014; PYRON y WIENS, 2011; FROST et al., 2006, FAIVOVITCH et al., 2005, BLACKBUM y WAKE, 2011; FROST, 2011 y bases de datos de Amphibian of the World, Amphibia Web, Anfibios de Ecuador, y BATRACHIA COLOMBIA. Adicionalmente las determinaciones fueron corroboradas con material de referencia de la Colección de Herpetología de la Universidad Tecnológica del Chocó (COLZOOCH-H).

Para la caracterización de uso de hábitat, se tuvo en cuenta la posición vertical o altura de la percha y tipo de sustrato en que se encontraban las especies, para lo cual se realizó registros de algunos datos ecológicos sugeridos por HEYER et al., (1994); de manera que se tuvo en cuenta dos variables: Posición vertical (altura desde el suelo o agua) tomada de forma cuantitativa así: suelo $(0 \mathrm{~cm})$, bajo $(1-49 \mathrm{~cm})$, medio (50 $149 \mathrm{~cm}$ ), alto (>149 cm) y el sustrato (sitio exacto en el que se capturó cada ejemplar) hojarascas, ramas, charcos, hojas, troncos, entre otros. Se documentó la información del estado de conservación, y endemismo de las según la UICN (2017).

Análisis de Datos: La abundancia relativa se determinó dividiendo el número de individuos colectados de cada especie sobre el total de individuos capturados en cada cobertura e igualmente se calculó el éxito de captura el cual revela la representatividad del esfuerzo de muestreo aplicado.

Se registró el número acumulado de especies versus el esfuerzo de muestreo para determinar la representatividad de los muestreos, para lo cual se utilizaron estimadores no paramétricos como $\mathrm{CHAO}$ 2 e ICE utilizando datos de presencia y ausencia, empleando el programa estadístico de Estímate (versión 6.0). Se calculó la frecuencia relativa como el número de apariciones por especies sobre el total de apariciones por áreas.

Respecto a la diversidad de especies, la diversidad alfa se trabajó mediante índices directos como la riqueza de especies (número de especies por área) y abundancia como el número de individuos por especies. Se utilizaron índices para trabajar con la abundancia proporcional, los índices de dominancia (Simpson), los índices de equidad (SHANNON-WEAVER y PIELOU) (BAEV y PENEV, 1995; VILLAREAL et al., 2004). La diversidad beta definida como la similitud de especies entre las zonas de muestreo (bosque primario y bosque secundario), se analizó mediante el coeficiente de similitud de Jaccard (MAGURRAN 1988).

Se realizó un análisis de varianza (ANOVA) de una vía, para comparar las áreas o sitios de muestreos con la finalidad de establecer diferencias significativas en la composición y la estructura de estas zonas; estos cálculos se realizaron con el programa PAST. Versión 1.15 (HAMMER y HARPER, 2003). Se realizó Chi-cuadrado para evaluar la preferencia de hábitat.

\section{RESULTADOS}

\section{Riqueza de Hílidos en zonas de bosque} pluvial tropical: Se registró un total de 137 individuos agrupados en cinco géneros y ocho especies; con un esfuerzo de muestreo de 240 horas/hombres, repartidas equitativamente en las coberturas estudiadas, para un éxito de captura de 0,57 ind. h/h. Al aplicar estimadores no paramétricos de riqueza se encontró que el estimador Chao 2, fue el que mejor predijo la riqueza de especies en ambos bosques, siendo el bosque secundario, el que registró el $100 \%$ de las especies estimadas, mientras que para bosque primario se pudo registrar el 93,98\% de las especies, e igualmente el estimador ICE predijo la totalidad de las especies registradas en el bosque secundario y el $66,75 \%$ para el bosque primario. Las curvas de acumulación de especies indican que los muestreos fueron significativos, ya que se pudo registrar casi la totalidad de las especies en cada uno de los bosques (Figura 2).

La familia Hylidae estuvo representada en cinco géneros (Boana, Dendropsophus, Smilisca, Agalychnis y Scinax) y ocho 

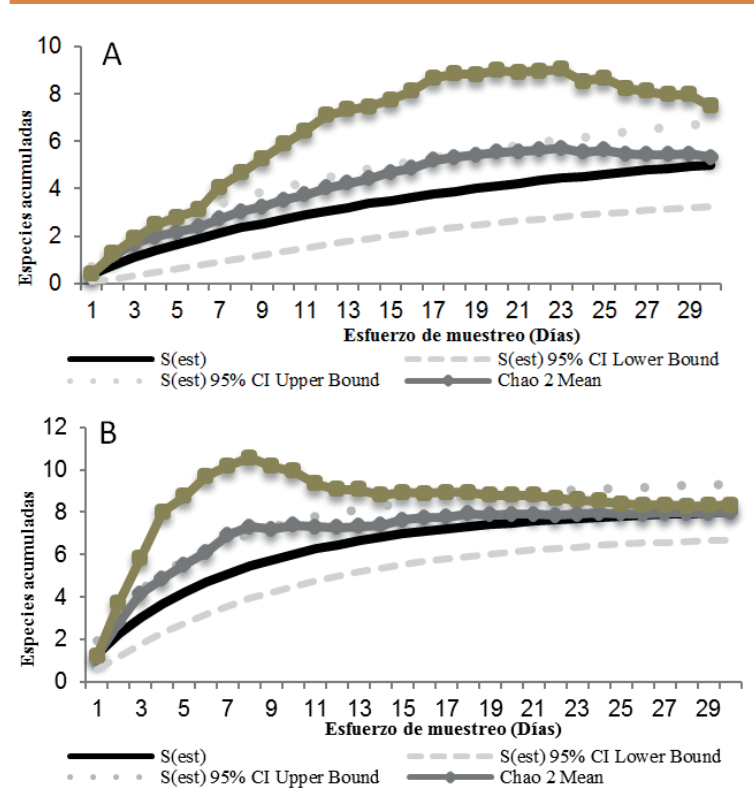

Figura 2. Curva de acumulación de especies de bosque primario (A) y secundario (B) en zonas de bosque pluvial tropical (bp-T) en el departamento del Chocó - Colombia. especies, los géneros mejor representado fueron el Dendropsophus, Scinax y Boana con dos especies cada uno, lo que equivale al $75 \%$ de las especies registradas (Tabla 2). Las ranas del género Dendropsophus son las más diversas de la familia Hylidae en Colombia con un total de 35 especies descritas hasta la fecha (ACOSTA-GALVIS y CUENTAS, 2016).

En el departamento del Chocó se registra un total de 22 especies de la familia Hylidae (ACOSTA-GALVIS y CUENTAS, 2016) de las cuales 13 se registran en la zona centro del departamento del Chocó distribuidas en cinco géneros los cuales son: Boana con cinco especies, Agalychnis con tres especies, Dendropsophus y Scinax con dos especies y Smilisca con una especie (Tabla 2).

En términos de abundancia, las especies mejor representadas fueron $B$. boans con el $48,9 \%$ seguida de $S$. phaeota con $24,82 \% A$. terranova $8,76 \% D$. phlebodes $5,84 \%$ y $S$. sugillatus $4,38 \%$

Tabla 2. Composición taxonómica de Hílidos en diferentes coberturas vegetales en zonas de bosque pluvial tropical del Departamento del Chocó- Colombia.

\begin{tabular}{|c|c|c|c|c|c|c|c|}
\hline \multirow{2}{*}{ Género } & \multirow{2}{*}{ Especies } & \multicolumn{2}{|c|}{ C. Vegetales } & \multicolumn{2}{|c|}{ Abundancias } & \multirow{2}{*}{\multicolumn{2}{|c|}{ UICN Fuente }} \\
\hline & & BP & BS & $(\mathbf{N})$ & $(\%)$ & & \\
\hline \multirow{3}{*}{ Agalychnis } & Agalychnis terranova (Rivera, Duarte, Rueda, Daza 2013) & 0 & 12 & 12 & 8,76 & NE & 3,5 \\
\hline & Agalychnis spurrelli (Boulenger 1913) & - & - & - & - & LC & 4 \\
\hline & Agalychnis psilopygion (Canatella 1980) & - & - & - & - & DD & 4 \\
\hline \multirow{2}{*}{ Dendrosophus } & Dendrosophus ebraccatus (Cope 1874) & 0 & 3 & 3 & 2,19 & LC & 2,5 \\
\hline & Dendrosophus phlebodes (Stenjer 1906) & 2 & 6 & 8 & 5,84 & LC & $2,4,5$ \\
\hline \multirow{5}{*}{ Boana } & Boana boans (Linnaeus 1758) & 27 & 40 & 67 & 48,9 & LC & 5,6 \\
\hline & Boana pellucens (Werner 1901) & - & - & - & - & LC & 2 \\
\hline & Boana rosenbergi (Boulenger 1898) & - & - & - & - & LC & 1 \\
\hline & Boana rubracyla (Cochran y Goin 1970) & - & - & - & - & LC & 2,4 \\
\hline & Boana rufitela (Fouquette 1961) & 0 & 3 & 3 & 2,19 & LC & 1,5 \\
\hline Smilisca & Smilisca phaeota (Cope 1862) & 2 & 32 & 34 & 24,8 & LC & $2,4,5$ \\
\hline \multirow{2}{*}{ Scinax } & Scinax sugillatus (Duellman 1973) & 2 & 4 & 6 & 4,38 & LC & $2,4,5$ \\
\hline & Scinax elaeochrus (Cope 1876) & 1 & 3 & 4 & 2,92 & LC & $2,5,6$ \\
\hline Total & & 34 & 103 & 137 & 100 & & \\
\hline
\end{tabular}

1. GRAJALES y PALACIOS (2000); 2. LYNCH y SUAREZ (2004); 3. Observación personal (Datos no publicados); 4 . PALACIOS y MORENO (2008); 5. ROA-P. y PALACIOS-A. (2016) 6. SALAS y MARTÍNEZ (2006). BP (Bosque Primario) BS (Bosque Secundario). 
Palacios-Rodriguez et al - Riqueza y distribución (Hylidae: Anura) en bosques pluvial tropical

(Tabla 2). Las demás especies presentaron abundancias relativamente bajas. En cuanto a la composición de especie por área de muestreo, el área con mayor número de especie fue la zona de bosque secundario con ocho (8) especies (100\%), mientras el bosque primario con cinco (5) especies $(62,5 \%)$. Las especies $D$. phlebodes, $B$. boans, S. sugillatus, $S$. elaeochrus y $S$. phaeota comparten ambas coberturas en contrastes con las especies $A$. terranova, $D$. ebraccatus, y $B$. rufitela, las cuales fueron observadas solo en bosques secundario (Tabla 2).

De acuerdo con la Unión Internacional para la Conservación de la Naturaleza (UICN) y el Convenio sobre el Comercio Internacional de Especies Amenazadas de Fauna y Flora (CITES), en este estudio no se registró ninguna especie incluida en alguna categoría de amenaza a nivel nacional, sin embargo, es indispensable proteger y conservar el hábitat de las especies de la familia Hylidae, puesto que son especies que cumplen un papel fundamental dentro de los ecosistemas, como indicadores, en la cadena alimenticia y otros servicios ecosistémicos que pueden ofrecer los anfibios.

\section{Estructura numérica de Hílidos en} zonas de bosque pluvial tropical: Teniendo en cuenta la estructura numérica de la comunidad de Hílidos en diferentes coberturas vegetales en zonas de bosque pluvial tropical, el bosque secundario presentó los mayores valores de equidad $\left(H^{`}: 1.58\right)$, en cuanto a la dominancia el bosque primario presento los mayores valores $\lambda=0.64$ (Tabla 3).

Tabla 3. Resultados generales de la estructura numérica de Hílidos en diferentes coberturas vegetales en zonas de bosque pluvial tropical del Departamento del Chocó- Colombia.

\begin{tabular}{ccc}
\hline Índices & $\begin{array}{c}\text { Bosque } \\
\text { Primario }\end{array}$ & $\begin{array}{c}\text { Bosque } \\
\text { Secundario }\end{array}$ \\
\hline Diversidad & 5 & 8 \\
Abundancia & 34 & 103 \\
Equidad & 0,79 & 1,58 \\
Dominancia & 0,64 & 0,27 \\
Riqueza (Margalef) & 1,13 & 1,51 \\
Equidad de Pielou(J) & 0,49 & 0,76 \\
\hline
\end{tabular}

Estos valores de dominancia, están soportados por $B$. boans y $S$. phaeota, ya que están bien representadas en las dos coberturas evaluadas, ya que son especies que presentaron abundancia debido a que las zonas de estudio presentan buena composición florística o disponibilidad vegetal para aquellas especies. No se encontró diferencia estadística significativa entre las coberturas objeto de estudio (ANOVA, $\mathrm{P}=0.1826$ ).

El porcentaje de similitud expresado por los índices cualitativos y cuantitativos, indica que existe un alto grado de semejanza entre las áreas estudiadas presentando mayores valores a nivel cuantitativo fue Morisita $(0,73 \%)$, por su parte, los coeficientes de Jaccard y Sorensen nos muestran que existe una mayor afinidad a nivel cualitativo $(0,63 \%)$.

Según los datos arrojados por la curva dominancia-diversidad, indican que la especie $B$. boans fue la más dominante para ambas coberturas. Para la zona de bosque secundario las especies más importante fueron $B$. boans y $S$. phaeota indicando así, la importancia de $B$. boans para las dos zonas estudiadas.

Uso de Hábitat de Hílidos en Zonas de Bosque Pluvial Tropical: Con respecto a la posición vertical, se encontró diferencias significativas $\left(X^{2}=118,53, P=1,34 E-15\right)$, los individuos fueron encontrados con mayor número en las posiciones suelo y medio con 55 y 43 respectivamente, seguido por alto y bajo con 26 y 13, respectivamente.

De acuerdo con el número de especies registradas en los diferentes sustratos estudiados, se encontró diferencia significativa $\left(X^{2}=132,4, P=1,66 \mathrm{E}-15\right)$, se evidencio que los sustratos rama, hojarasca y hoja, fueron los más representativos.

Al analizar la distribución estructural del hábitat ocupado por las especies de Hílidos, se observó que este grupo de individuos presentó mayor frecuencia de ocurrencia en la posición vertical suelo con $(n=56)$ y ubicados en el sustrato charco $(n=38)$, seguido de la posición vertical medio y alto 
en sustratos rama para ambos; y por último la posición vertical baja con pocos individuos en el sustrato tronco, rama y hoja. (Tabla 4).

Tabla 4. Distribución estructural del microhábitat de las ranas arborícolas de la familia Hylidae en diferentes coberturas vegetales en zonas de bosque pluvial tropical del Departamento del ChocóColombia.

\begin{tabular}{ccccccc}
\hline $\begin{array}{c}\text { Sustrato/ } \\
\text { P. Vertical }\end{array}$ & Suelo Bajo Medio & Alto & Total & \% \\
\hline Hojarasca & 18 & & & & 18 & 13,14 \\
Tronco & 5 & & & 5 & 3,65 \\
Rama & & 4 & 28 & 23 & 55 & 40,15 \\
Charco & 38 & & & & 38 & 27,74 \\
Hoja & & 4 & 17 & & 21 & 15,33 \\
\hline Total & $\mathbf{5 6}$ & $\mathbf{1 3}$ & $\mathbf{4 5}$ & $\mathbf{2 3}$ & $\mathbf{1 3 7}$ & $\mathbf{1 0 0}$ \\
\hline
\end{tabular}

\section{DISCUSIÓN}

Este comportamiento de las curvas puede atribuirse a la eficiencia en los muestreos y/o a la homogeneidad del hábitat, además, los anfibios son más ricos y diversos en bosques secundarios y maduros, debido a su anatomía y morfología, lo cual los hace dependientes de la humedad que estos hábitats les ofrece (VARGAS y BOLAÑOS, 1999b).

La alta representatividad de Hílidos en este estudio, en cuanto al número de especies, es significativa al ser comparados con las investigaciones que existen para la zona centro del departamento del Chocó, debido a que se registraron ocho especies de Hílidos, lo cual representa el $61,53 \%$ de las 13 especies registradas para esta zona.

El hecho de que $B$. boans sea la especie más representativa puede estar relacionado con su ecología, ya que es una especie generalista que se relaciona con cuerpos de aguas temporales y bosques riparios aledaños a bosques secos; estas especies, es común observarla en cuerpos de agua artificiales y temporales presentes en zonas urbanas o en áreas con alta perturbación antrópica (CUENTAS et al., 2002).

También podría deberse a la disponibilidad de numerosos árboles cercanos a cuerpos de agua ya que en este estudio esta especie presentó numerosos registros en ramas de árboles y arbustos cercanos a cuerpos de agua; además, la alta representatividad de esta especie es significativa, debido a que fue registrada en las dos coberturas vegetales (bosque primario y bosque secundario). GRAJALES, et al. (2003) hacen referencia a la presencia de especies de Hílidos en zonas intervenidas en el corregimiento de Salero, argumentando que esta presencia es atribuida a que, estas zonas o ambientes ofrecen la suficiente estructura vegetal para permitir la sobrevivencia de un considerable número de especies.

En cuanto a los resultados obtenidos en la comparación de especies en las coberturas evaluadas ESTUPIÑAN y GALATTI (1999) afirman que especies de la familia Hylidae son potenciales indicadores de ambientes con algún grado de alteración de la cobertura vegetal natural, ya que son tolerantes a las transformaciones de la misma luego de actividades antrópicas. En cuanto a la exclusividad, al igual que VARGAS y BOLAÑOS (1999b) creemos que las especies registradas únicamente en bosques secundarios posiblemente están presentes en bosques primarios y no fueron observadas debido a la poca abundancia de las especies y limitaciones de observación.

El comportamiento de cada una de estas especies se debe a que $D$. ebraccatus habita en bosque húmedo tropical primario y secundario, y donde la mayor parte de la cobertura ha sido destruida; igualmente, la época reproductiva es prolongada y coincide con la época de mayor lluvia (DUELLMAN, 1970). B. rufitela, esta especie se reproduce en estanques poco profundos, charcos y áreas fangosas, pudiendo tolerar algún grado de perturbación antrópica (CUENTAS et al., 2002) y $A$. terranova es una especie nocturna arbórea, asociada a vegetación cerca de cuerpos de aguas como tanques artifíciales en bosque primario, secundario y áreas alteradas tales como potreros adyacentes a bosques (RIVERA-CORREA et al., 2013).

Lo anterior permite inferir que, en el caso particular de los anuros, el hábitat juega un factor importante para determinar la composición de especies que ocurren en un sitio determinado, ya que el principal factor 
Palacios-Rodriguez et al - Riqueza y distribución (Hylidae: Anura) en bosques pluvial tropical

que amenaza la supervivencia de los anfibios es la pérdida y el deterioro del hábitat a causa de actividades antropogénicas (YOUNG et al., 2004), el alto grado de competencia y depredación, un individuo puede escoger un sitio diferente con menos recursos óptimos (KRAUSMAN, 1999).

El hecho de que el bosque primario presentara mayores valores de dominancia y el bosque secundario de equidad, podrían deberse a que, las especies de Hílidos registradas en este estudio son tolerantes a los habitas perturbados, ya que son especies que se asocian fácilmente a la vegetación baja en bosques secundarios

Los valores de equidad y dominancia están relacionados con la representatividad de especies como $B$. boans y S. phaeota, que se distribuyeron de una forma casi equitativa en las zonas estudiadas ya que estas coberturas evaluadas les ofrecen suficiente cobertura vegetal que les sirve de soporte al hábitat que conserva condiciones para camuflaje, refugio y alimentación, favoreciendo a el grupo de los Hílidos. Los anfibios han sido considerados excelentes modelos para establecer el nivel de deterioro de los hábitats y ecosistemas del mundo (PECHMANN y WILBUR 1994; STEBBINS y COHEN, 1995; RUEDA-ALMONACID et al., 2004).

El hecho de que $B$. boans sea dominante y que junto a $S$. phaeota estén bien representadas en las coberturas evaluadas se atribuye a que las áreas de estudio, se caracterizan por presentar una gran variedad de hábitats que favorecen a esta especie al presentar una gran adaptabilidad y tolerancia a las alteraciones antrópicas producidas en sus hábitats (MOLINA et al., 2000).

Respecto a la posición vertical de las especies de Hílidos registrados en este estudio, los resultados concuerdan en su mayoría con los obtenidos por VARGAS et al. (2000), en dos localidades para el Pacífico colombiano quienes demostraron que los Hílidos por ser generalistas frecuentan los cuatro rangos de altura, dentro de la estructura de la vegetación. Los Hílidos usan sustratos y posiciones verticales variables con respecto a la actividad diaria, resaltando la importancia que la mayoría de las especies contienen individuos arbóreos y terrestres, además, activos entre los 0 y $150 \mathrm{~cm}$ del suelo. La representatividad de la posición suelo en este estudio, concuerda con ROMERO-M et al. (2008), quien sostiene que a pesar de que algunas especies de anfibios son típicas del dosel, para el caso de los anfibios los estratos mejor aprovechados son el sotobosque y el estrato basal.

en cuanto al sustrato o sitio de percha de especies de hílidos, los resultados obtenidos concuerdan en gran medida con muñozguerrero et al. (2007) quienes afirman que algunos hílidos usan las ramas más altas de los arbustos y árboles de la orilla, aspecto que podría estar ocurriendo con las especies de registradas en este estudio ya que los sitios de muestro presentaban cobertura de gran tamaño.

CÁCERES-A. y URBINA-C. (2009) realizaron un estudio en tres coberturas vegetales en el piedemonte llanero, departamento del Meta, y los sustratos hojas y ramas fueron los de preferencia para los Hílidos, registrados en ese estudio, al igual que FAIVOVITCH et al. (2005), quienes sostienen que la variedad de ambientes satisface los requerimientos de hábitat de las especies de anfibios, minimizando la competencia intra e interespecífica. KRAUSMAN (1999) afirma que para el caso de los anuros la selección de hábitat puede ser por cobertura, calidad y cantidad de forrajeo, sitios de percha o refugio, procesos que pueden variar estacionalmente

En la distribución estructural del microhábitat indica que las especies de Hílidos asisten o concurren todas las posiciones verticales y los sustratos lo que concuerda con un estudio en Anchicayá, Pacífico colombiano VARGAS y CASTRO (1999) indican que algunas especies de Hílidos frecuentan todos los tipos de sustratos y posición vertical seleccionados.

\section{Agradecimientos}

Agradecemos enteramente al grupo de investigación en Herpetología de la Universidad tecnológica del Chocó por el financiamiento de este proyecto, su acompañamiento y guía en campo e identificación de los ejemplares colectados, además, de permitir el acceso a la colección. 


\section{REFERENCIAS}

ACOSTA-GALVIS A.R.; CUENTAS, D. 2016. Lista de los Anfibios de Colombia: Referencia en linea V.05.2015.0 (Citado 21 oct 2016). Página web accesible en http://www. batrachia.com.

ANGULO, A. 2002. Anfibios y paradojas: Perspectivas sobre la diversidad y las poblaciones de Anfibios. Ecología Aplicada 1(1): 105-109.

ANÓN, 1982C. Filling, capping, and labelling developments. Int. Bottler Packer 56.

BAEV, P.V.; PENEV, L.P. 1995. Biodiversity: Program for calculating biological diversity parameters, Similarity niche overlap, and cluster analysis. Version 5. I. persot Sofia - Moscow.

BLACKBUM, D.C.; WAKE, D.B. 2011. Clase Amphibia Gray, 1825. Págs. 38-54. En: Zhang-Q, Z. (ed.). Biodiversidad animal: un resumen de alto nivel de la clasificación y el reconocimiento de la riqueza taxonómica. Zootaxa.

BLAUSTEIN, A.R.; WAKE, D.B.; SOUSA, W.A. 1994. Amphibian Declines: Judging stability, Persistende, and susceptibility of populations to local and global extinctions. Conservation Biology 8(1): 60-71.

CÁCERES, A.S.; URBINA, C.J. 2009. Ensamblajes de Anuros de sistemas productivos y bosques en el Piedemonte Llanero, Departamento del Meta, Colombia. Caldasia 31(1):175-194.

CUENTAS, D.; BORJA, R.; LYNCH, J.D.; RENJIFO; J.M. 2002. Anuros del departamento del Atlántico y norte de Bolívar. Universidad del Atlántico. CRA. Barranquilla, Colombia.

CRUMP, M.; SCOTT, N. 1994. Visual encounter surveys Págs. 80-87. En: Heyer R.W., Donnelly, M.; McDiarmis, R.; Hayek, L.; Foster, M. (eds), Measuring and Monitoring, Biological Diversity: Standard methods for amphibians Smithsonian institution Press, Washington.

CUATRECASAS, J. 1958. Aspectos de la vegetación natural de Colombia. Revista de la Academia Colombiana de Ciencias 10(40): 221-268.

DUELLMAN, W.E. 1970. Hylid Frogs of Middle America. Monograph Museum Natural History University of Kansas 1: 1-753.

ESTUPIÑÁN, R.; GALATTI, U. 1999. La fauna anura en áreas con diferentes grados de intervención antrópica de la Amazonia Oriental Brasileña. Revista de la Academia Colombiana de Ciencias Exactas, Físicas y Naturales. Suplemento Especial 23: 275-286.

FAIVOVICH, J., HADDAD, C.F.B.; GARCIA, P.C.O. ; FROST, D.R. ; CAMPBELL, J.A. ; WHEELER, W.C. 2005. Systematic review of the frog family Hylidae, with special reference to Hylinae: phylogenetic analysis and taxonomic revision. Bulletin of the AMERICAN Museum of Natural History 204:1-240.

FORERO, E.; GENTRY, A.H. 1989. Lista anotada de las plantas del departamento del Chocó, Colombia. Biblioteca J.J. Triana 10 Instituto de Ciencias Naturales, Universidad Nacional de Colombia. 
Palacios-Rodriguez et al - Riqueza y distribución (Hylidae: Anura) en bosques pluvial tropical

FROST, D.R. 2011. Amphibian Species of the World: an online reference. Version 5.5. Electronic Database accessible at http://research.amnh. org/vz/herpetology/amphibia/. American Museum of Natural History, New York, USA.

FROST, D., GRANT, T.; FAIVOVICH, J.; BAIN, R.; HASS, A.; HADDAD, C.; CHANNING, R.A.; WIKINSON, M.; DONNELLY, S.; RAXWORTHY, CH.; CAMPBELL, J.; BLOTTO, B.; MOLLER, P.; DREWES, R.; NAUSSBAN, R.; LYNCH, J.D.; GREEN, D. Y WHEELER, W.C. 2006. The Amphibians Tree of Life. Bulletin of the American Museum of Natural History Central Park West At.

GRAJALES, D.; PALACIOS, H. 2000. Composición y estructura de la comunidad de anuros en áreas con diferentes grados de intervención antrópica de Salero, Unión Panamericana, Chocó. Trabajo de Grado. Facultad de Ciencias Básicas. Universidad Tecnológica del Chocó, Quibdó, Chocó.

GRAJALES, D.; PALACIOS, H.; ECHEVERRY, C.; GIL, N. 2003. Composición y estructura de la comunidad de anuros en áreas con diferentes grados de intervención antrópica de Salero, Unión Panamericana. Págs. 91-102. En: García, F.; Ramos, Y.; Palacios, J.; Arroyo, J.; Mena, A.; González, M. (eds.). Diversidad Biológica de un Bosque Pluvial Tropical (bp-T) Salero. Editorial Guadalupe Ltda.

HALLIDAY, T.; ADLER, K. 2007. La gran enciclopedia de los anfibios y reptiles. Libsa, Madrid.

HAMMER, D.; HARPER, D.A.T. 2003. Past- Versión 1.15 h http: Fol. Uio No/o Hammer past.

HANKEN, J. 1999. Why are there so many new amphibian species when amphibians are declining? Trends in Ecology and Evolution 14(1): 7-8.

HEYER, W.R.; DONNELLY, M.A.; MCDIARMID, R.W.; HAYEK, L.C.; FOSTER, M.S. 1994. Measuring, and monitoring biological diversity, standard methods for amphibians, Smithsonian inst. Press. Editorial Universitaria de la Patagonia, Argentina.

KIESECKER, J.M.; BLAUSTEIN, A.R.; MILLER, C.L. 2001. Transfer of a Pathogen from fish to amphibian. Conservation Biology 4(15): 1064- 070.

KNUTSON, M.; SAUER, J.R.; OLSEN, D.A.; MOSSMAN, M.J.; HEMESATH, L.M.; LANNOO, M.J. 1999. Effects of landscape composition and wetland fragmentation on frog and toad abundance and species richness in Iowa and Wisconsin, USA. Conservation Biology 13(6): 1437-1446.

KNUTSON, M.; SAUER, J.R.; OLSEN, D.A. ; MOSSMAN, M.J.; HEMESATH L.M. ; LANNOO, M.J. 2000. Landscape associations of Frog and Toad species in Iowa and Wisconsin, USA. J. Iowa Academy Scienty 107(3): 134-145.

KRAUSMAN, P. 1999. Some basic principles of habitat use. Págs. 85-90. En: Launchbaugh, K.I.; Sanders, K.D.; Moslev, J.C. (Eds.). Grazing behaviour of livestock and wildlife. Idaho Forest, Wildlife y Range. University of Idaho, Moscow.

LIPS, K.R.; BURROWES, P.A.; MENDELSON, J.R.; PARRA-OLEA, G. 2005a. Amphibian declines in Latin America: Widespread population declines, extinctions, and impacts. Biotropica $37(2): 163-165$.

LYNCH, J.D.; SUÁREZ-MAYORGA, A. 2004. Catálogo de anfibios en el Chocó Biogeográfico. Pp, 654-668. En Rangel (ed.) Colombia Diversidad Biótica IV, El Chocó Biogeográfico/ Costa Pacífica. Volumen I. Bogotá: Universidad Nacional de Colombia. 
MAGURRAN, A.E. 1988. Ecological diversity and its measurement. Princeton University Press, New Jersey.

MENDOZA, J.S. 2014. Aportes Para La Identificación de Las Ranas Gladiadoras del Género Hyspsiboas (WAGLER, 1830); (ANURA: HYLIDAE), Presente en las Tierras Bajas del Caribe Colombiano. Revista. Colombiana de Ciencia Animal 6(1): 55- 69.

MOLINA, L.L.A.; AGUDELO, A.; PEÑA, R.M.J.; CUARTAS, C.C.A.; ROLDAN, P.F.; PARRA, C.J.D. 2000. Inventario de Flora y Fauna de la Reserva la Guarcana, Municipio de Buriticá Antioquia. Marzo 29. Corantioquia.n/ohammar/ past.

MUÑOZ-GUERRERO, J.; SERRANO, V.H.; RAMÍREZ-PINILLA, M.P. 2007. Uso de microhábitat, dieta y tiempo de actividad en cuatro especies simpátricas de ranas hílidas neotropicales (Anura: Hylidae). Caldasia 29(2): 413-425.

OSORNO-MUÑOZ, M. 1999. Evaluación del Efecto de Borde para poblaciones de Eleutherodactylus (Amphibia: Anura: Leptodactylidae), frente a corredores de servidumbre en diferente estado de regeneración en dos bosques intervenidos por líneas de transmisión eléctrica de alta tensión. Revista de la Academia Colombiana de Ciencias Exactas, Físicas y Naturales (Sumplemento especial) 23: 347-356.

PÁEZ, V.P.; BRIAN, C.B.; ESTRADA, J.J.; ORTEGA, A.M.; DAZA, J.M.; GUTIÉRREZ-C, P.D. 2002. Guía de campo de algunas especies de anfibios y reptiles de Antioquia. Universidad Nacional de Colombia, Conciencias, Universidad de Antioquia, Medellín.

PALACIOS-RODRÍGUEZ, P.; RENGIFO-MOSQUERA, J.T.; LYNCH, J.D. 2016. First record and distribution extension of Agalychnis terranova (Rivera-Correa, Duarte-Cubides, RuedaAlmonacid y Daza, 2013) (Anura: Hylidae: Phyllomedusinae) in the Pacific lowlands of Colombia. Revista Herpetology Notes. 9: 109-111.

PALACIOS, J.; MORENO, H. 2008. Diversidad de anuros en tres áreas con diferentes tipos de intervención antrópica en Certegui, Chocó - Colombia. Trabajo de Grado. Facultad de Ciencias Básicas. Universidad Tecnológica del Chocó, Quibdó, Chocó.

PALACIOS, R.P.; RENGIFO, M.J.; ECHAVARRÍA, R.J. 2013a. Primer reporte de Dendropsophus bogerti (Anura: Hylidae: Hylinae) en el departamento del Chocó, Colombia. Revista de Biodiversidad Neotropical 3(2): 127-30.

PALACIOS, R.P.; QUESADA, M.K.; RENGIFO, M.J. 2013b. Nuevo registro y ampliación de distribución geográfica para Agalychnis psilopygion (Anura: Hylidae: Phyllomedusinae) en el Chocó Biogeográfico de Colombia. Revista de Biodiversidad Neotropical 3(2): 123-6.

PECHMANN, J.H.; SCOUT, D.E.; SEMLITSCH, R.D.; CALDWELL, J.P.; VITT, L.J.; GIBBONS, J.W. 1991. Declining amphibian populations: The problem of separating human impacts from natural fluctuations. Science 53(5022): 892-895.

PECHMANN, J.K.H.; WILBUR, H.M. 1994. Putting declining amphibian populations in perspective: Natural fluctuations and human impacts. Herpetológica 50: $65-84$.

POUNDS, J.A.; FOGDEN, M.P.L.; CAMPBELL, J.H. 1999. Biological responses to climate change on a tropical mountain. Nature 398: 611-615.

POUNDS, J.A.; PUSCHENDORF, R. 2004. Clouded futures. Nature 427:107-109. 
Palacios-Rodriguez et al - Riqueza y distribución (Hylidae: Anura) en bosques pluvial tropical

POVEDA, M.C.; ROJAS, C.A.; RUEDAS, A.; RANGEL, J.O. 2004. El Chocó biogeográfico: Ambiente físico. Págs. 1-21. En: Rangel, J.O. (Ed). Colombia Diversidad Biótica IV: El Chocó biogeográfico/ Costa Pacífica. Universidad Nacional de Colombia, Bogotá D.C.

PYRON, R.A.; WIENS, J.J. 2011. A large-scale phylogeny of Amphibia including over 2800 species, and a revised classification of advanced frogs, salamanders, and caecilians. Molecular Phylogenetics and Evolution 61(2): 543-583.

RAFINESQUE, C.S. 1814. Fine del Prodromo d'Erpetologia Siciliana. Specchio delle Scienze, o, Giornale Enciclopedico di Sicilia 2: 102-104.

RANGEL-CH, J.O.; LOWY-C, P. 1993. Tipos de vegetación y rasgos fitogeográficos. En: P. Leyva (ed.). Colombia Pacífico: 182-198. Fondo FEN - Colombia. Bogotá.

RENJIFO, J.M.; LUDBERG, G. 1999. Guía de Campo; Anfibios y Reptiles de Urrá. Editorial Colina. Medellín, Colombia.

RIVERA-CORREA, M.; DUARTE-CUBIDES, F.; RUEDA-ALMONACID, J.V.; DAZA, J.M. 2013. A new red-eyed treefrog of Agalychnis (Anura: Hylidae: Phyllomedusinae) from middle Magdalena River valley of Colombia with comments on its phylogenetic position. Zootaxa 3636 (1): 85-100.

ROMERO-MARTÍNEZ, H.J.; VIDAL-PASTRANA, C.; LYNCH, J.D.; DUEÑAS, P.R. 2008. Estudio preliminar de la fauna Amphibia en el Cerro Murrucucú, PNN-Paramillo y zona amortiguadora, Tierralta, Córdoba, Colombia. Revista Caldasia 30 (1): 205-225.

RUEDA-ALMONACID, J.V.; LYNCH, J.D.; AMÉZQUITA, A. (Eds.). 2004. Libro rojo de anfibios de Colombia. Serie Libros Rojos de Especies Amenazadas de Colombia. Conservación Internacional Colombia, Instituto de Ciencias Naturales-Universidad Nacional, Ministerio del Medio Ambiente. Bogotá, Colombia.

SALAS, O.; MARTÍNEZ, R. 2006. Diversidad de anuros en la cuenca del rio Cabí, departamento del Chocó - Colombia. Trabajo de Grado. Facultad de Ciencias Básicas. Universidad Tecnológica del Chocó, Quibdó, Chocó.

SMITH, J.; SABOGAL, C. ; JONG, W. ; KAIMOWITZ, D. 1997. Bosque secundario como recurso para el desarrollo rural y la conservación ambiental en los trópicos de América Tropical. Taller internacional sobre el estado actual y potencial de manejo y desarrollo del bosque secundario tropical en América Latina. (2000, Pucallpa. PE). Memorias.

STEBBINS, R.C.; COHEN, N.W. 1995. A natural history of amphibians. Princeton University Press, New Jersey.

STUART, S.N.; CHANSON, J.S.; COX, N.A.; YOUNG, B.E. 2006. El estado global de los Anfibios. Págs. 19-42. En: Angulo, A.; Rueda-Almonacid, J.V.; Rodríguez-Mahecha, J.V.; La Marca, E. (Eds.). Técnicas de Inventario y Monitoreo para los Anfibios de la Región Tropical Andina. Conservación Internacional. Serie Manuales de Campo N02. Panamericana Formas e Impresiones S.A., Bogotá D.C.

SUÁREZ-NAVARRO, A.E.; HURTADO-PEÑA, G. ; CARVAJAL-LEMUS, F.J.; RODRÍGUEZ-BAUQUERO, J.E.; RODRÍGUEZ-SOTO, R. 1984. Mapa de Bosques de Colombia: diversidad de anolis del Chocó, Colombia. 63 memoria explicativa. Instituto Geográfico "Agustín Codazzi" - Instituto Nacional de Desarrollo de los Recursos Naturales y del Ambiente - Corporación Nacional de Investigación y Fomento Forestal, Bogotá, Colombia. 
URBINA-CARDONA, J.N.; CARVAJAL-COGOLLO, J.E. 2008. Patrones de Diversidad y Composición de Reptiles en Fragmentos de Bosque Seco Tropical en Córdoba, Colombia. Tropical Conservation Science 1(4): 397-416.

VARGAS, F.; BOLAÑOS, M. 1999b. Anfibios y Reptiles en Hábitats Perturbados de la Selva Lluviosa Tropical en el Bajo Anchicayá, Pacífico colombiano. Revista de la Academia Colombiana de Ciencias 23: 499-511.

VARGAS, F.; CASTRO, F. 1999. Distribución y preferencias de micro-hábitat en anuros (Amphibia) en bosque maduro y áreas perturbadas en Anchicayá, Pacífico colombiano. Caldasia 21: 95-109.

VARGAS, S.F.; BOLAÑOS, M.E.; BERRIO-B, H. 2000. Notas sobre la ecología reproductiva de Agalychnis spurrelli (Anura: Hylidae) en una población de Anchicayá, Pacífico colombiano. Revista Academia Ciencias Exactas, Físicas y Naturales 24 (90):85-99.

VILLARREAL, H.; ÁlVAREZ, M.; CÓRDOBA, S.; ESCOBAR, F.; FAGUA, G.; GAST, F.; MENDOZA, H.; OSPINA, M.; UMAÑA, A.M. 2004. Manual de métodos para el desarrollo de inventarios de biodiversidad. Instituto Alexander von Humboldt. Bogotá, Colombia.

YOUNG, B.E.; STUART, J.S.; CHANSON, S.N.; COX, N.A.; BOUCHER, T.M. 2004. Joyas que están desapareciendo: El estado de los anfibios en el Nuevo Mundo. NatureServe, Arlington, Virginia:1- 55 